\title{
Question and answer sequences in Garrwa talk
}

\author{
ROD GARDNER
}

Griffith Institute for Educational Research and Faculty of Education, Griffith University

For question-answer sequences in Australian Aboriginal talk, it has been claimed that answers are not necessarily a required response. This would contrast with findings reported in recent cross-linguistic work on such sequences (Stivers, Enfield and Levinson, 2010; Stivers et al., 2009). In a corpus of 62 question sequences from conversations in two Garrwa communities on the west side of Gulf of Carpentaria in northern Australia, 34 questions were answered, and a further 12 dealt with the question in some other way. 16 received no response in the proximally subsequent talk. Whilst most of these questions were answered, the offset time between question and response was long compared to the Stivers et al. (2009) studies. There was also a higher rate of non-answers and non-responses. For some cases of non-responses, contingent factors easily explained the lack, but in a few the reasons were not so apparent. It is argued that a significant factor in the relatively long silence between question and answer, and the relatively high rate of non-answers or nonresponses, is that the parties in the talk spend much of the time in 'continuing states of incipient talk', rather than in tightly focused and temporally bound conversation, which may help to account for the apparent relaxation of gap minimization and response mobilization. 


\section{Questions, Answers, Universals and Delay}

Australian linguistic and anthropological field workers have regularly claimed that there are differences in various aspects of communicative style between Indigenous Australians and Anglo Australians. One of these claimed differences is that questions as requests for information are treated differently. Referring to questions addressed to Aboriginal witnesses and defendants in legal settings, Eades (2007:286) states:

The interview genre is typically a one-sided information exchange, where one party asks questions and the other provides answers. ...nonAboriginal interviewers often reported that they experienced difficulty in eliciting information from Aboriginal interviewees, while Aboriginal interviewees often reported feeling rushed, pressured, and unable to take the time they need to answer questions properly.

Eades (1985) suggests that unlike in western conversations, in Aboriginal talk there is no obligation to give information, and thus there is 'no obligation on the addressee to answer a question' (p. 98). As an example, she cites a case in which, 'under questioning by the lawyers, [an Aboriginal woman being questioned] provided very little information. The lawyers asked her questions, and she appeared unable to provide answers' (Eades, 2007:289).

Eades (1992:27-8) makes it clear that many information-seeking questions addressed to Aboriginal addressees are in fact answered, but these are of particular types: 
Direct questions are used in some settings, particularly to find out background details, e.g. “Where’s he from?” However, in situations where Aboriginal people want to find out what they consider to be significant or certain personal information, they do not use direct questions. It is important for Aboriginal people to respect the privacy of others, and not to embarrass someone by putting them "on the spot”.

In other words, information seeking does occur, but in certain more sensitive situations it is much less direct. Furthermore, the questioner contributes what they already know about the topic of the question, thereby restricting the scope of the sought information (Eades 1993). This entails that information is often exchanged rather than sought, so there is less of a unidirectional transmission of information from answerer to questioner. One would thus expect to find fewer direct questions in Aboriginal interactions than in Anglo ones, and those that are used would be about 'light' or non-substantial matters.

Drawing on his extensive linguistic field work experience with Aboriginal Australians, Walsh was also struck by the ways in which questions are dealt with, and like Eades found that questions were regularly not answered: 'Numerous questions are asked. And sometimes the answer does not come back as fast as you might expect. Indeed, often no answer comes back at all' (Walsh 1997:4). He goes on to ponder (p. 4-5):

Is this so-called ‘delayed’ reaction an isolated phenomenon? Far from it. I have spoken to plenty of people who have worked in Aboriginal Australia over the years and in fact nearly all of them agree that exactly the same thing has happened to them. And I have found that it need not just be a week. In some instances the answer might come back several years later but not with any explicit linkage to 
the original question. Quite puzzling for the Anglo, but apparently not at all puzzling for the Aboriginal person.

We have, then, the apparent regular absence of what 'ought' to follow a question, namely an answer. Schegloff (1996) remarks on the non-occurrence of such a second pair part that 'one is warranted in speaking of its absence or avoidance in the strongest sense, because its relevance has already been introduced by the participants - by their very launching of its deployment' (p. 195). According to observations such as those reported by Eades and Walsh, the Aboriginal answerer may not necessarily be party, it seems, to an agreement that an answer should follow a question, at least not immediately. Does this mean that Indigenous Australians' interactional style and practices present a challenge to the proposals within Conversation Analysis that interactional phenomena such as turntaking, or the strong links between canonical adjacency pair types such as questions and answers, are likely to turn out to be human universals (e.g. Schegloff 2006, Levinson 2006)?

One of the more or less explicit thrusts of Conversation Analytic work is that the underlying machinery of conversation is likely to be universal to all human societies and groups (e.g. Sidnell 2001; Stivers et al 2009). ${ }^{\mathrm{i}}$ Amongst these suggested universals is that actions are paired, such as invitations and accepts/declines, offers and accepts/rejects, or questions and answers. Schegloff (1968: 1083) puts it this way:

Given the first, the second is expectable; upon its occurrence it can be seen to be a second item to the first; upon its nonoccurrence it can be seen to be officially absent - all this provided by the occurrence of the first item. 
There is thus an obligation on the second speaker to perform a particular response type that is fitted to the type of the first utterance, and this is to be done contiguously to the first - or as close to contiguously as possible. By implication we can expect that answers will follow questions, and on the few occasions that they do not come, some kind of action by the question recipient that accounts for their absence could occur, or some reason detectable in the data should account for the absence of an answer, such as the recipient being engrossed in some other activity. Is this what we find in Aboriginal conversations?

Answering is only one way of dealing with a question, so not answering a question does not mean that the question recipient is not dealing in some way with the question. This may, for example, be something similar to 'I don’t know'; it may be confirming a suggestion, rather than simply answering; or it may be initiating repair on the question. A non-conforming response (cf. Raymond, 2003) is, of course, still a response, which is different from 'no response at all' that Eades and Walsh observe.

Stivers et al. (2009), consider question-answer sequences in a range of languages and cultures from advanced industrial (North America, Europe, East Asia), through agricultural (South America, Papua New Guinea), to hunter-gatherer groups (Africa). Their work endorses the view that it is a universal of human interaction that questions project answers as next actions - that is, they make answers conditionally relevant next actions to questions, and that answers or relevant responses are provided continuously and promptly in most cases in these languages (cf. Schegloff 1968; Schegloff \& Sacks 1973). As Stivers \& Rossano (2010) say, ‘...in the context of requests for information recipients orient to themselves as accountable for producing an answer either by providing an answer or by accounting for nonanswer responses' (p. 7). Their universalist position is that this is likely to be the case for all languages and all cultures (see also Stivers et al. 2009). 
Further evidence for the universal nature of question-answer adjacency pairs is presented in a collection of papers in the Journal of Pragmatics, coming out of the Max Planck crosscultural project referred to above (Stivers, Enfield \& Levinson, 2010). Amongst the issues discussed in each of the papers in this collection is the nature of the response to the question, which they coded into three categories: whether the question was answered (typeconforming), or received a non-answering response (non-type-conforming), or received no response at all. They found variation across the languages in the proportions of these three types of response, and in some cases provided accounts for this variation. Across all 10 languages, they found that most questions were answered, that most of the rest received non-answer responses. Only a minority was not answered, in some cases an almost vanishingly small number.

In some respects, the studies revealed strong variation across languages. In Dutch only 4\% of questions got no response at all (Englert, 2010), and in US English the figure is 5\% (Stivers, 2010), a figure that the author feels may be inflated, as some of these questions are answered eventually, which is not captured in the coding system used in this collection.

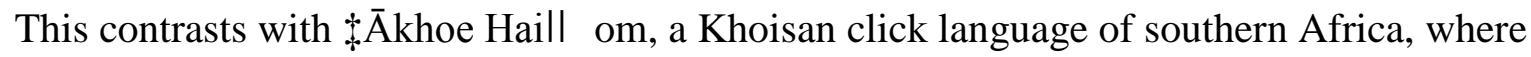
Hoymann (2010) found 23.2\% of questions received no response, and in Lao a full quarter of all questions were found to receive no response (Enfield, 2010). Hoymann noted that lack of response was particularly high for questions that do not select next speaker (more than a third of these received no response), but even when next speaker was selected, $15 \%$, or nearly one in seven, were not answered. She accounts for this as possibly being concomitant with the egalitarian, hunter-gatherer life style of the $₫ \bar{A}$ khoe, with 'no institutionalized social or political hierarchy’ (Hoymann, 2010:2737), and where equal access to resources and reciprocity are highly valued.

Some of the other authors in this collection also attempt to account for the occurrence of 
no response to questions. Levinson (2010) suggests for Yélî Dnye, a language spoken on Rossel Island in Papua New Guinea, that 'we found that lack of gaze by the recipient is a good predictor of lack of response...suggesting that a major reason for no response is disengagement or lack of attention’ (p. 2751). 13\% of questions received no response, verbal or visual in this Yélî Dnye data set. Brown (2010) examined Tzeltal data (a Mayan language spoken in south-eastern Mexico). She accounts for the $7 \%$ of questions in her data that do not get responses as being due to factors such as the questioner (e.g. a child) having no rights to the floor, the addressee displaying hesitancy in responding to questions that are, for example, offers (i.e. not requests for information), or because the question is directed to the questioner him- or herself. But '[o]verwhelmingly, Tzeltal questions are treated as expecting an answer in the next turn, and an answer is in fact usually supplied' (p. 2639). Many of the other papers make only passing reference to the questions that are not responded to - which is understandable in a set of studies that are considering aggregates of coded categories.

Another phenomenon considered in the Stivers et al. (2009) collection is response offset times: that is, how long it takes for a response to be produced. In these Max Planck studies, the languages with the three highest mean offset times are Danish, $\neq \bar{A}$ khoe Haill om and Lao, at $0.47,0.42$ and 0.42 seconds approximately and respectively. The shortest are Japanese, Tzeltal and Yélî Dnye, at $<0.01,0.07$ and 0.07 respectively. So silences of close to half a second on average are relatively common in the first three languages, whereas responses come close to or even before completion of the question to which they are responding in the latter three. One can thus conclude that across the 10 languages, the time lapse before a response to a question, whether an answer or a non-answering response, tends to come within half a second of completion of the question. 
If it can be demonstrated that Aboriginal Australians, as Eades and Walsh suggest, do not necessarily treat questions as requiring an answer, or at least some orientation to the absence of an answer, and if there are long response offset times when they are answered, then there needs to be some account within CA for the practice for treating first parts of adjacency pairs as not requiring a second part under certain circumstances. There also needs to be an account for non-minimization of gap between first and second pair parts under certain circumstances, and more widely a reconsideration of the notion of conditional relevance. Questions and answers are an excellent test case, as questions are canonical first pair parts.

Stivers \& Rossano (2010) argue that first pair parts are designed very strongly for a response by the presence of at least one of the following design features:

- Interrogative syntax (or question morpheme/particle)

- Interrogative prosody (such as rising terminal intonation)

- Epistemic skewing towards the recipient (recipient is deemed to have the knowledge sought in the question/request for information)

- Speaker gaze directed towards recipient

Stivers and Rossano propose these conditions mobilize a second pair part response to all first pair parts that they examined in their American English and Italian database (not only questions - in fact the main focus of their paper is on assessments, noticings and announcements). They found that if one of these conditions in present, then the first part of the adjacency pair will elicit a response. They say that actions such as requests, offers and questions are more canonical, and claim that the presence of these mobilizers is part of what makes them canonical, and thus more likely to elicit a response. 
Their claims, however, have met with some resistance. Schegloff (2010), for one, argues against stepping 'downward on the ladder of granularity’ to establish 'mobilizing' conditions (p. 46), suggesting instead that overall structural organization might be a more useful analytic focus for explaining the lack of responses on certain conversational occasions, citing the contrast between 'continuously sustained talk' (which has been the focus of most CA studies) and ‘continuing states of incipient talk’ (Schegloff \& Sacks, 1973). The latter is more likely to be found amongst 'members of a household in their living rooms, employees who share an office, passengers together in an automobile' (pp. 324-5). On such occasions it is not necessary to close a conversation every time the talk lapses, or to re-open it with greetings when talk starts up again. What makes such occasions different from 'continuously sustained talk' is that the conversation is open-ended and not co-terminous with the occasion, in the way that, for instance, telephone conversations or interviews are. Couper-Kuhlen (2010) makes a related point in her critique of Stivers and Rossano (2010). She appeals to Goffman's distinction between 'focused' and 'nonfocused' gatherings: the former include tennis, dancing and everyday conversations (Kendon, 1988), which 'maintain single focus of cognitive and visual attention' (Goffman, 1963:89). In 'nonfocused' gatherings, on the other hand, there is a lack of 'tightly organized exchange of doings...of a common “clock”' (Couper-Kuhlen, 2010, p. 35). In 'continuing states of incipient talk', there may then be less urgency to produce second pair parts.

In the current paper, the focus is on question-answer sequences in five ordinary conversations conducted out of doors and in public in Garrwa communities near the Gulf of Carpentaria country in northern Australia. They involve five elderly Aboriginal women, and a regular stream of passers-by. In these conversations, the women use both Garrwa and Kriol when talking to each other, and English or Kriol mainly (though some Garrwa) when speaking with younger community members. This study builds on work by Mushin and 
Gardner into turn-taking practices (Gardner \& Mushin 2007, Mushin \& Gardner 2009) in everyday Aboriginal conversation, in which overlapping talk and silence were studied. I use a relatively small corpus of five conversations to investigate their ways of answering each other's questions. 62 questions were located in the corpus of a little over one hour of closely transcribed conversations. The vast majority were WH-questions, ${ }^{\text {ii }}$ with eight polar and three tag questions, ${ }^{\text {iii }} 34$ of the questions were answered, 12 received non-answer responses, and 16 were not answered at all. This means that $55 \%$ of questions were answered, and $45 \%$ were not, which is a lower percentage of answered questions than any in the Stivers et al. (2009) study, ${ }^{\text {iv }}$ in which Korean, at $64 \%$, was the lowest. Of those questions that were answered, most were answered immediately (or after relatively short offsets of up to about half a second), but many others were responded to after substantial delays.

After presenting question-answer sequences that can be considered canonical from a Western standpoint, the focus will be on a set of delayed and partial responses, and a striking case of a complete lack of a response.

\section{Question and Answer Sequences in Garrwa Conversations}

As noted above, most of the 62 questions in the collection were responded to directly and at least relatively promptly. The average silence length following a question was 1.15 seconds. If one takes only questions that get an answer or a relevant response, the average silence is 0.75 seconds: about one third of a second longer than the longest found in the Stivers et al. (2009) study (Danish, $\ddagger \bar{A}$ khoe Haill om and Lao). Whilst this metric suggests that these Garrwa speakers delay their responses more than any of those in the Stivers et al. 
study, there is no evidence that they treat these gaps as problematic, and thus that the answers are, for them, 'delayed'.

The rate of non-response, at $26 \%$, is about the same as Lao, which had the highest rate of non-response of the 10 languages in the Stivers et al. (2009) study. Of the 'no response' question sequences, there appear no obvious grounds for not answering in a few of them in the Garrwa corpus, even with video data evidence.

The first set of extracts presents some of the cases in which a question receives a relatively prompt answer. In the first, Tina and Ellen ${ }^{\mathrm{v}}$ are talking on the veranda of a house before the linguistic fieldwork of elicitation commences. They are catching up on the overnight news in their township. They can see the main street of the town and part of the pub from where they are sitting. ${ }^{\mathrm{vi}}$

(1) Borroloola 3: 20-8-03:3

3

4 Ellen: Hhuh

5

6 Tina: Wạ:jbarr' nand' murdu. what-BARRI that mad.one

$$
\text { Q1-> Where's that mad one }
$$
$(0.4)$

8 Ellen: Im e:re-u la pob. he here LOC pub A1-> He's here at the pub $(0.5)$

10 Tina: - HEHH !=

11 Ellen: $\quad{ }^{\star} \underline{N}:$ : ${ }^{\star}$ ayiba walkin'; 


\section{here walking \\ A1-> Walking (around) here}

$(0.9)$

13 Tina: Where e:?

Q2-> Where is he?

14

$(0.5)$

15 Ellen: La- pub-.

LOC pub

\section{A2-> At the pub}

There are two question-answer sequences in this extract. In the first, Tina asks where 'the mad one' (a man who had been in a fight the previous evening) was. Ellen's answer comes relatively promptly, after 0.4 seconds (line 8 ), and she follows this with some incremental information that he is 'walking around here' - which suggests that he is within their field of sight. Tina's next question appears not to be a repetition of the first question because of a possible lack of hearing, despite its similar form, but a question about whether she should be able to see him. Ellen's answer is a repeat that he's at the pub - and thus perhaps out of their line of sight.

Next I present an array of question-answer sequences in which the questions receives a direct answer. In the first the answer comes within 0.2 seconds of the question.

(2) Garrwa2-9-10-03:234

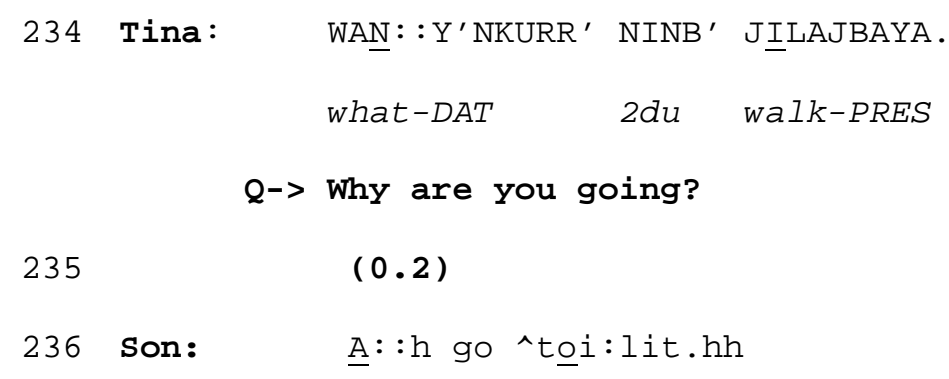




\section{A-> I'm going to the toilet}

In the next one the question initiates a repair, and it receives its answer within 0.3 seconds.

(3) Garrwa2-20-8-03:91

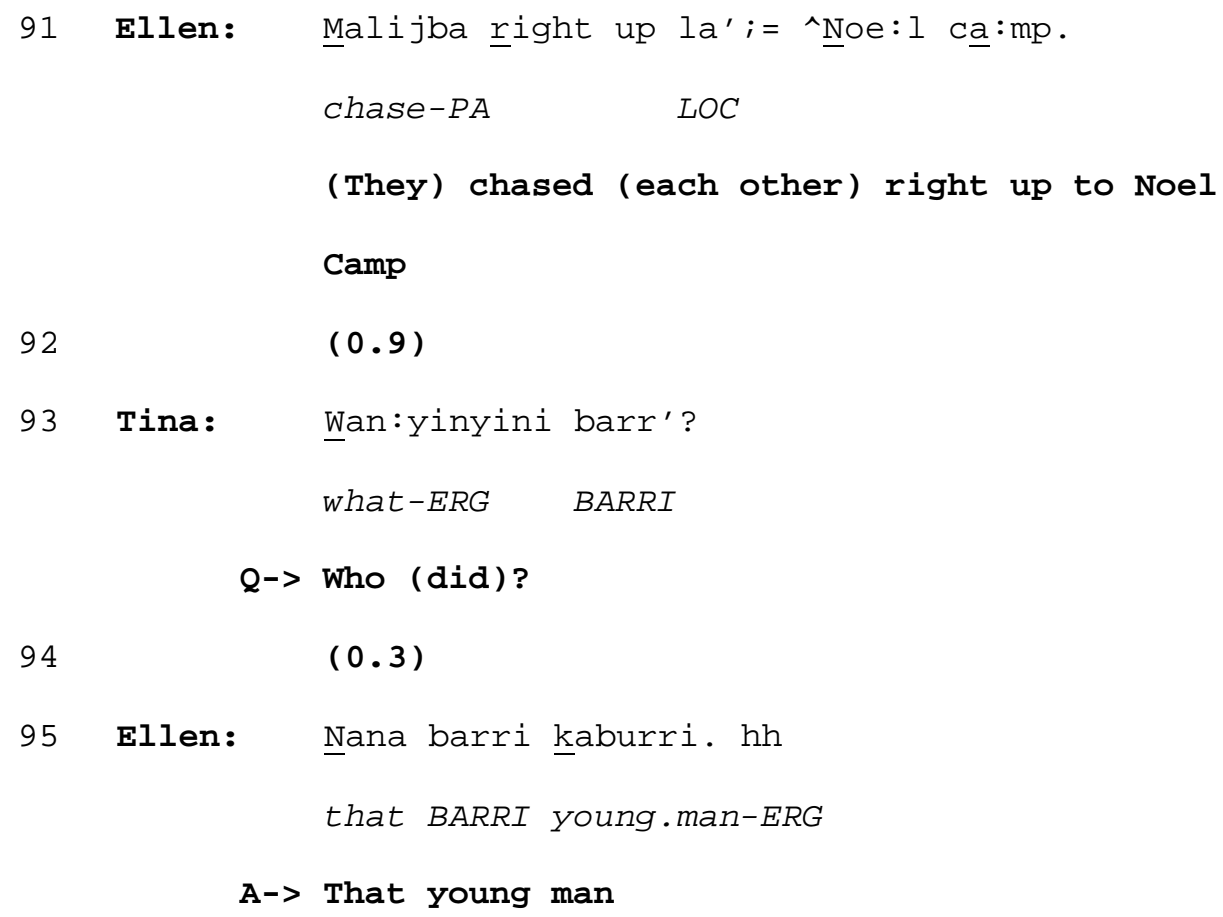

These two questions can be seen as ‘background' questions - direct and not significant or particularly personal (cf. Eades 1992), so the kinds of questions that Eades would consider of the type that is likely to receive a straightforward answer.

Extract (4) is an example of an immediate answer where one might expect it would be delayed in an Anglo-conversation, as Sonny does not know the answer to the question - the response in non-conforming, in Raymond's (2003) sense.

(4) Garrwa2-9-10-03:242

$$
\begin{aligned}
242 \text { Ellen: [> } & \text { Ngala wanj'wa: where da- murdu. } \\
& \text { ConJ where where that mad.one }
\end{aligned}
$$




\section{Q-> (So) where, where's that mad one}

243

$(0.2)$

244 Son:

$>$ Ah $d^{\prime}$ know. whereim;<dey look'n f'r im init $i<$

A-> I don't know where he is, they're looking for

him, aren't they?

Many of the questions in the data set are answered in this prompt manner: almost half of the answers or relevant responses occur within half a second. Regularly answers, however, follow quite significant silences, often where there is little evidence that there is a problem with providing the answer, such as it being disagreeing or dispreferred, or in a domain of privacy or speaking rights. All five of the elderly women in these conversations are about the same age, and have known each other since childhood. There are no kin relations that require indirection in addressing each other. Whilst these silences following the questions may not be seen as excessive, because of their regularity in these conversations, they do provide some evidence of a relaxation of the requirement to minimize gaps (Sacks, Schegloff \& Jefferson, 1974). They also seem not to fully comply with the adjacency pair operation whereby 'a given sequence will...be composed of an utterance that is a first pair part produced by one speaker directly followed by the production by a different speaker of an utterance which is (a) a second pair part, and (b) is from the same pair type as the first utterance in the sequence is a member of' (Schegloff \& Sacks, 1973:296 - emphasis added). Note the issue is only about whether the SPP follows directly or not. This may be because these conversations are open-ended, and not bounded by a clear beginning or ending of the occasion.

The remainder of the examples are taken from the 'Porch’ conversation between Kate, Hilda and Daphne, with other parties coming and going. For much of the time the old women are on their own. They had already been sitting on the Porch for over an hour at the 
beginning of the transcription from which these examples are taken. Hilda not being present from the beginning of the filming at the start of the first hour. During the second hour, they complain a few times about being tired and hungry.

In extract (5), Daphne is asking a question (970) about a detail of a story Kate is telling. There is mutual gaze between her and Kate. They disengage gaze at the end of the question, and 0.7 seconds later Kate answers.

(5) Porch:2.4:595

970 Daph: $\quad[\mathrm{Na}$

Na wanyi- (. ) > wanyimba barri yalu.<

PART what - what-do $\quad 3 p 1$

Q-> And what did they do?

$971 \quad(0.7)$

972 Kate: Wudumba yil' yal' nanau: ¿

Get $\quad H A B \quad 3 p l$ that

A-> They used to get it

In extract (6), Kate is asking for a more exact location for a place called Kangaroo Creek. She and Kate have eye contact, with Kate's head almost at right angles to her torso to maintain the gaze. There is still a considerable silence of 1.1 seconds before Hilda answers, and there is no lip pursing or other gesture during this silence. She does, though, start to turn her head away from Kate - apparently in the direction of 'the north side of the road' about 0.2 seconds before she starts speaking.

(6) Porch:2.4:1384

\section{Hilda: Kangaroo $\underline{\text { Creek.hh }}$}

1385

$(0.2)$

1386 Kate: $\quad$ Yang:kawa. 


\section{Q-> Whereabouts}

1387 (1.1)

1388 Hilda:

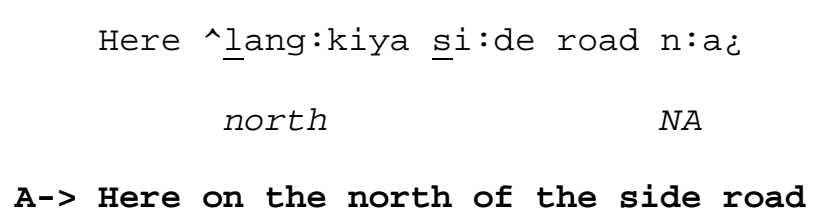

In extract (7), which occurs earlier in the same storytelling by Kate, the answer may be heavily deictic, but it is nevertheless a relevant and fitting response to the question. Daphne and Kate have eye contact, with Kate withdrawing gaze about half way through her answer, which follows a silence of 1.4 seconds. During this silence and her answer, Kate is fiddling with the collar of her shirt.

(7) Porch:2.4:1004

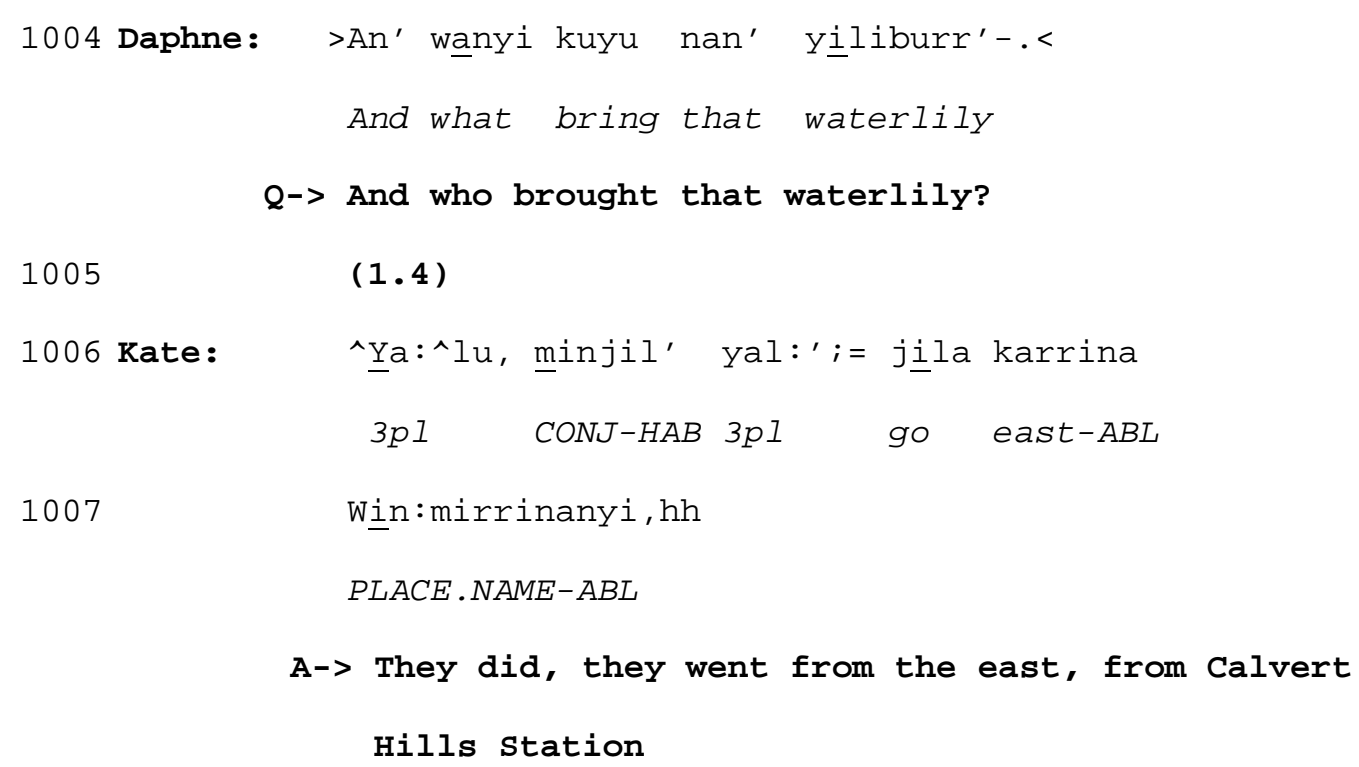

All of the questions in extracts (5) to (7) are answered with some silence between question and answer, but without any apparent trouble - and none appear to be about sensitive or potentially restricted or particularly personal topics. There is eye contact in all three examples, so no lack of engagement. The only possible distraction is Kate fiddling 
with her collar in extract (7), though the fact that she is still fiddling with it whilst she is answering demonstrates that this has not been strongly distracting.

Before turning to some examples in which the sequences deviate to a greater or lesser extent from those above, one further extract, (8), shows full engagement by all four participants in providing direct and timely answers to a series of question. Three questions are asked, and all receive multiple answers. The three question-answer sequences interlock without any apparent disruption to the talk. Someone had killed a kangaroo, which was being cooked somewhere in the community. Kate asks where it is, but does not select an answerer; a second question (one of the eight polar questions in the data set) asks how it is being cooked, and a third question is concerned with where the kangaroo has come from.

(8) Porch:2.4:611

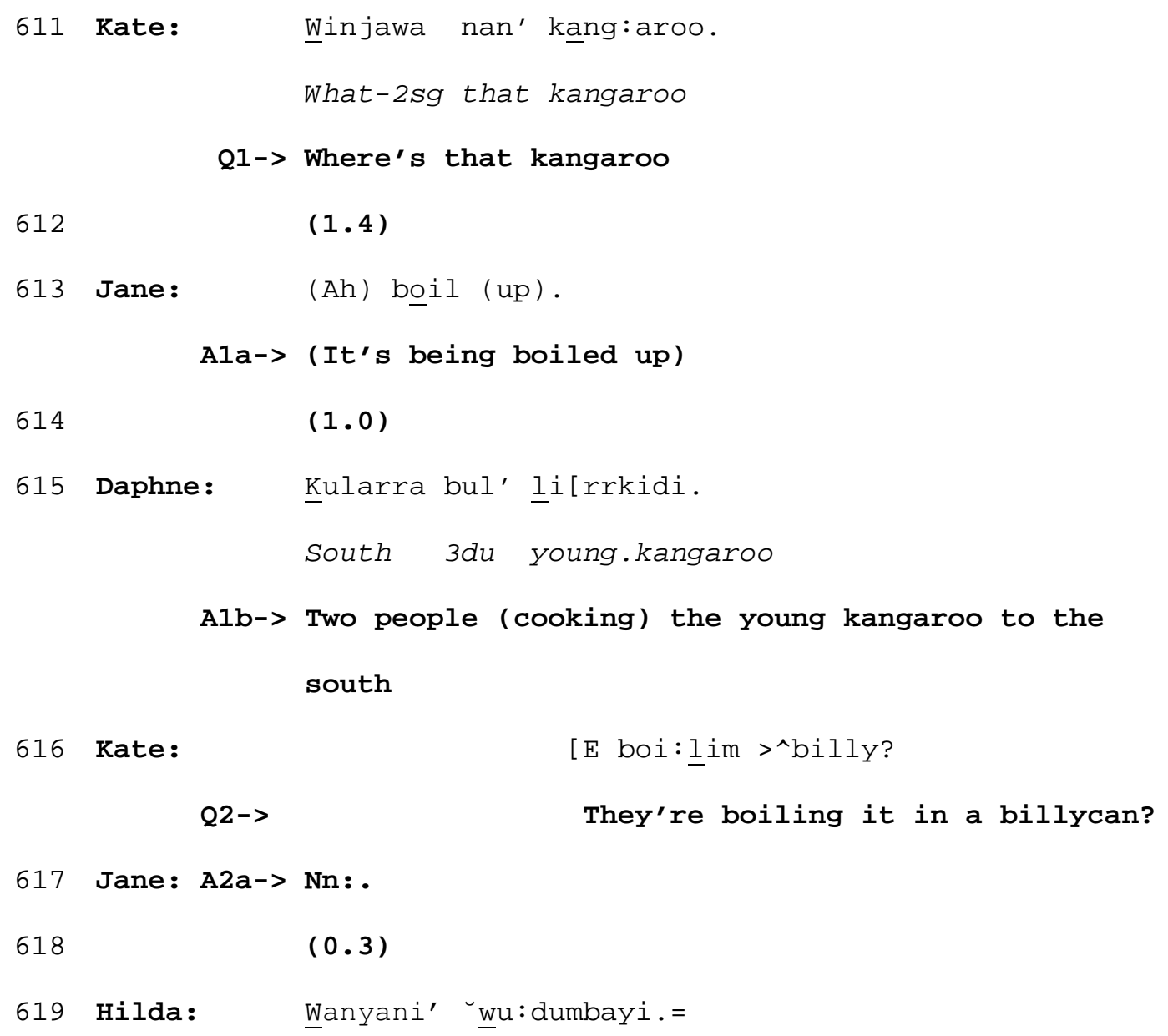




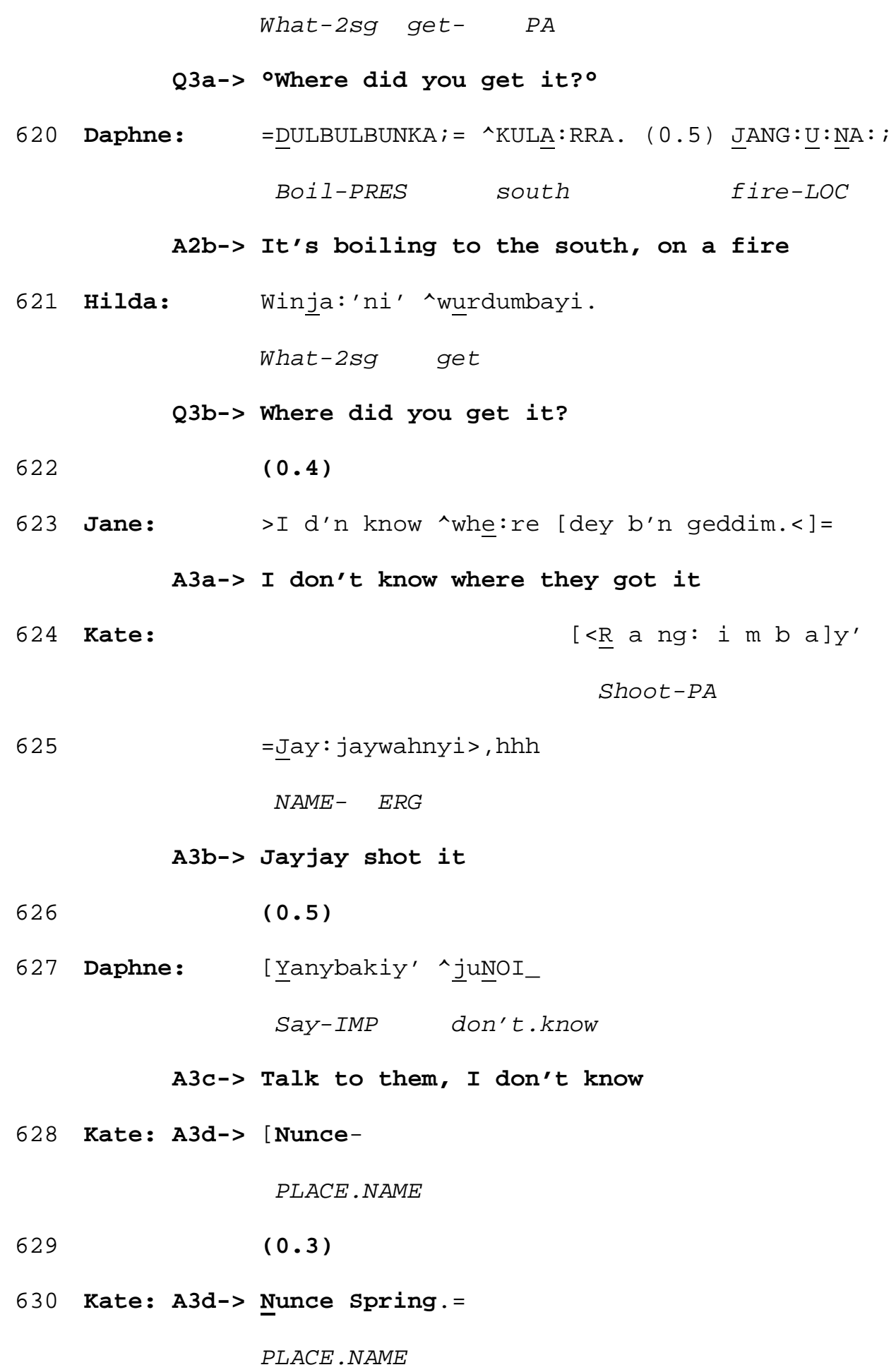

Kate, in her question about the location of the kangaroo (611), selects Jane as recipient by gaze - turning her head up and to the left towards her standing by the porch. Jane responds after what appears to be a quite long silence of 1.4 seconds, but during this silence she 'lip points', that is, she turns her head in the direction of the place where the kangaroo is being 
boiled and purses her lips, before she verbally answers. Thus there is in effect no delay in the answer, as the first part is produced non-verbally. A second answer comes from Daphne (615) , after a further gap of a second, in which she adds to the first answer (two people are cooking the kangaroo, and she verbalizes the direction). Whilst this second answer is being produced, Kate asks Jane (616), using a declarative polar question, whether it is being cooked in a billycan (a kind of kettle), and receives an affirming answer.

Hilda next asks a third question about where the kangaroo had been caught (619), but before an answer comes, Daphne provides an incremental answer to the second question (620), adding that the kangaroo is being cooked on a fire. Hilda is sitting behind the other two old women, out of their line of sight, and her question is spoken softly, which may help account for the lack of an immediate answer, and she repeats her question (621), asking where she got the kangaroo, gazing in the direction of Jane. The second version of the question is louder, and the intonation is more strongly marked. After a short silence, Jane, who has begun to turn away, gives a non-type conforming answer, declaring she does not know the answer. Kate, however, does know the answer, and just after 'I don’t know where' (623) - which is where it has become apparent that Jane will not answer - Kate provides an answer (625), although it is, strictly speaking, about who shot the animal, not where it was shot. A short time after this, Daphne, the third potential answerer, suggests Hilda ask someone else (627), as she does not know the answer (627). Finally, Kate comes in with the fourth response to Hilda's question (628), simultaneously with Daphne, then with a restart and full answer (630). This time she provides a fully type-conforming answer, the name of the place where the Kangaroo was shot.

In this sequence the four participants in these question-answer sequences display as well as they might their orientation to answering questions. One speaker self-selects as the first responder, and then others come in with subsequent responses that provide answers where 
the first responder could not, or to increment and complement the prior answers with further information. In this extract, the four women are producing three interactionally complex and interlocking question-answer sequences in a manner that - in terms of turntaking, silence between first and second pair parts, and producing actions fitted to the sequence - are structurally and interactionally very much like what has been presented in the extensive literature examining in detail such sequences, including for other cultures, notably in the recent Max Planck worked cited above.

I shall turn now to three final examples in which no answer is forthcoming. In some of the other instances in the data set in which there is no response to a question, there is, for example, a deliberate ignoring of the question by a recalcitrant child, unanswered questions being followed after a gap by repeats of the questions, or the questioner supplying an answer herself. In the first two of the final set of extracts below, the lack of an answer can be accounted for through other local interactional contingencies. Only the final example is virtually (but not entirely) bereft of any evidence that the speakers are orienting to the question.

In extract (9), the three women are sitting on the porch, whilst a dog is persistently barking loudly.

(9) Porch:2.4:75

$\begin{array}{llll}75 & \text { Dog: } & ((\text { bark-bark })) & - \\ 76 & & (0.5) & \mid \\ 77 & \text { Dog: } & ((\text { bark-bark-bark-bark-bark })) \\ 78 & & (0.4) & \\ 79 & \text { Dog: } & {[((\text { bark - bark - bark }))} \\ 80 & & (1.0)\end{array}$


81 Daphne: [Barriwa

\section{(Finish)}

82

$(1.5)$

83 Hilda: Wanya nani wandu(wab)a

What that barking

Q-> What's that barking?

84

$(0.4)$

85

Dog:

( (bark-bark-bark))

$(1.5)$

86

$(0.2)$

87 Kate: Badadajb' yalu king[karrinya,

Come-PL $3 p I \quad$ up-ABL

A1-> They're coming up

88

((Someone or something approaching?))

89 Dog:

[( (bark-bark-bark) )

$9 \odot$ Hilda: Wakanya

Pig

Pig

91

$(1.0)$

92

Dog:

(barks)

$(1.8)$

93 Kate: Kingka[rrinya: bulanya $\mathrm{hh}[\mathrm{sh}$

$$
\begin{array}{ccc}
u p-A B L & 3 d u-A C C & \text { [ } \\
\text { A2-> Two (coming) from up (there) [ }
\end{array}
$$

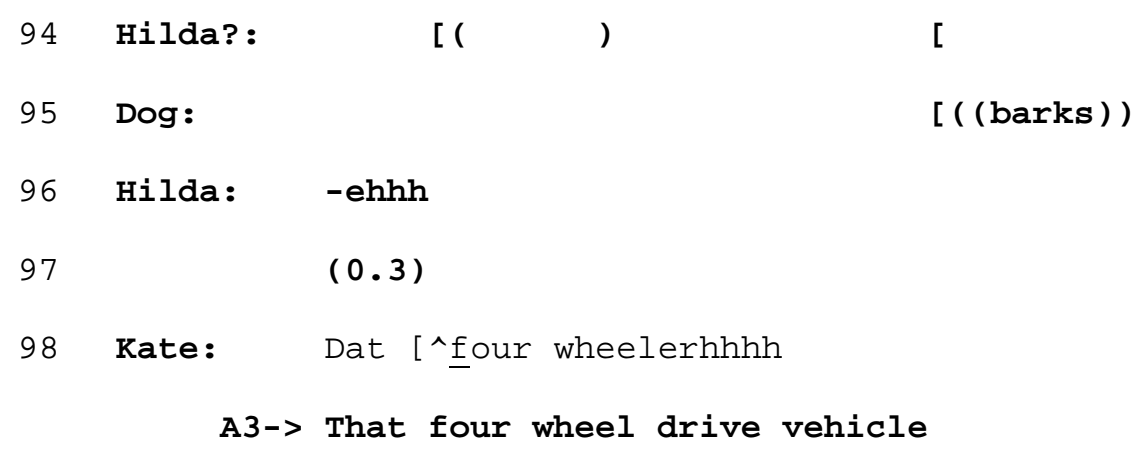


103 Kate: Four wheeler comin' [from dere

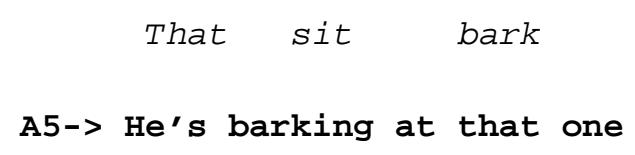

In line 83, Hilda asks, 'What’s that barking?' After 1.5 seconds, Kate provides a response ('They're coming up', line 87) that appears to be indexical of the cause of the barking - the approach of someone or something. She elucidates in line 93 that 'two are coming up from there'. At line 98, she looks up and away from the porch as she says, 'That four wheeler', which can be taken as a further elaboration of the answer, and in lines 103 and 115, as the four wheel drive vehicle approaches, she moves towards the clearest answers, that the dog is barking at that vehicle. This question is one to which, one can assume, Kate first provided an approximate answer; then, as the cause of the barking reveals itself, through the persistent barking and the approach of the vehicle, she elaborates on the answer incrementally. Thus the apparent lack of an answer can be explained by no answer being readily or immediately available. When a full answer becomes available, Kate provides it. The answers are supplied in a leisurely fashion, with considerable gaps of silence between 
the various answers, and 26 seconds between the question (83) and the fifth answer (115). There is also some talk on other topics intervening.

In extract (10), Daphne and Kate combine forces to ask Don where he had seen another man who had been in town that morning. The question is first asked by Daphne in lines 499/502, spoken in overlap with the targeted recipient's talk in line 500 (Don is looking through the viewfinder of the camera at this point).

(10) Porch:2.6:497

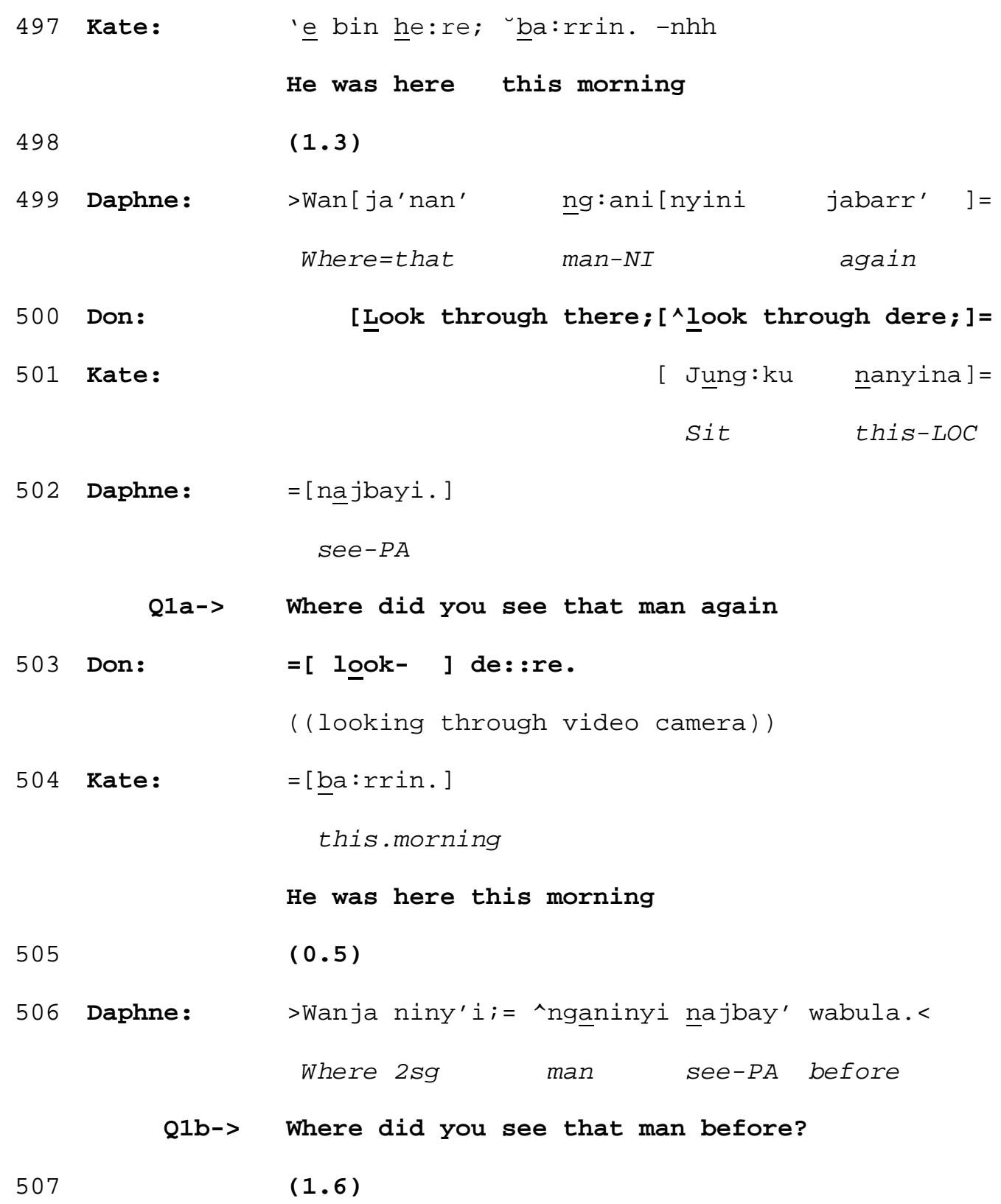


Daphne: $\quad>$ Wanja ni $\left[n^{\prime}\right.$ najba;= nganinyi] ${ }^{\circ}$ nga:buji: ${ }^{\circ}<$ Where $2 s g$ see man ((FaMoBr $)$ )

Q1c-> Where did you see the man, the great uncle?

509 Kate:

$$
\begin{aligned}
& \text { [(Wanj'wa) ju:no??] } \\
& \text { (Where) I don't know }
\end{aligned}
$$

510

$(0.4)$

511 Kate:

R: onnie wanyi.

Ronnie-ERG

Ronnie did

512

$(0.8)$

513 Don:

(Wha: rah.)

$$
\text { Resp1-> (Where?) }
$$

514

$(0.8)$

515

Daphne:

>Nganinyinyi najba 'rri;= kingka la

$$
\text { Man-ERG see BARRI up LOC }
$$

516

Blacksfella-; S: - Spring:; $=$ ^ngarri?

$$
\text { Blackfella spring ngarri }
$$

Q1d-> The man (saw him) up at Blackfella Spring, didn't he?

517

(1.1)

518 Kate:

Junkg'wa ^k:a`ja n’na w:anka;= nganinyi:?

Sit-WA many (that) alive man

Big mob of men still living there

519

(1.5)

520 Kate:

[>Kabu ^y:an]ybakiy' nana'kanyi; =

$$
\text { Look, talk-IMP that-DAT }
$$

521 Daphne: [( )]

522 Kate: =bardibardinyi,

old. woman-DAT

Look, talk to that one, to the old woman 


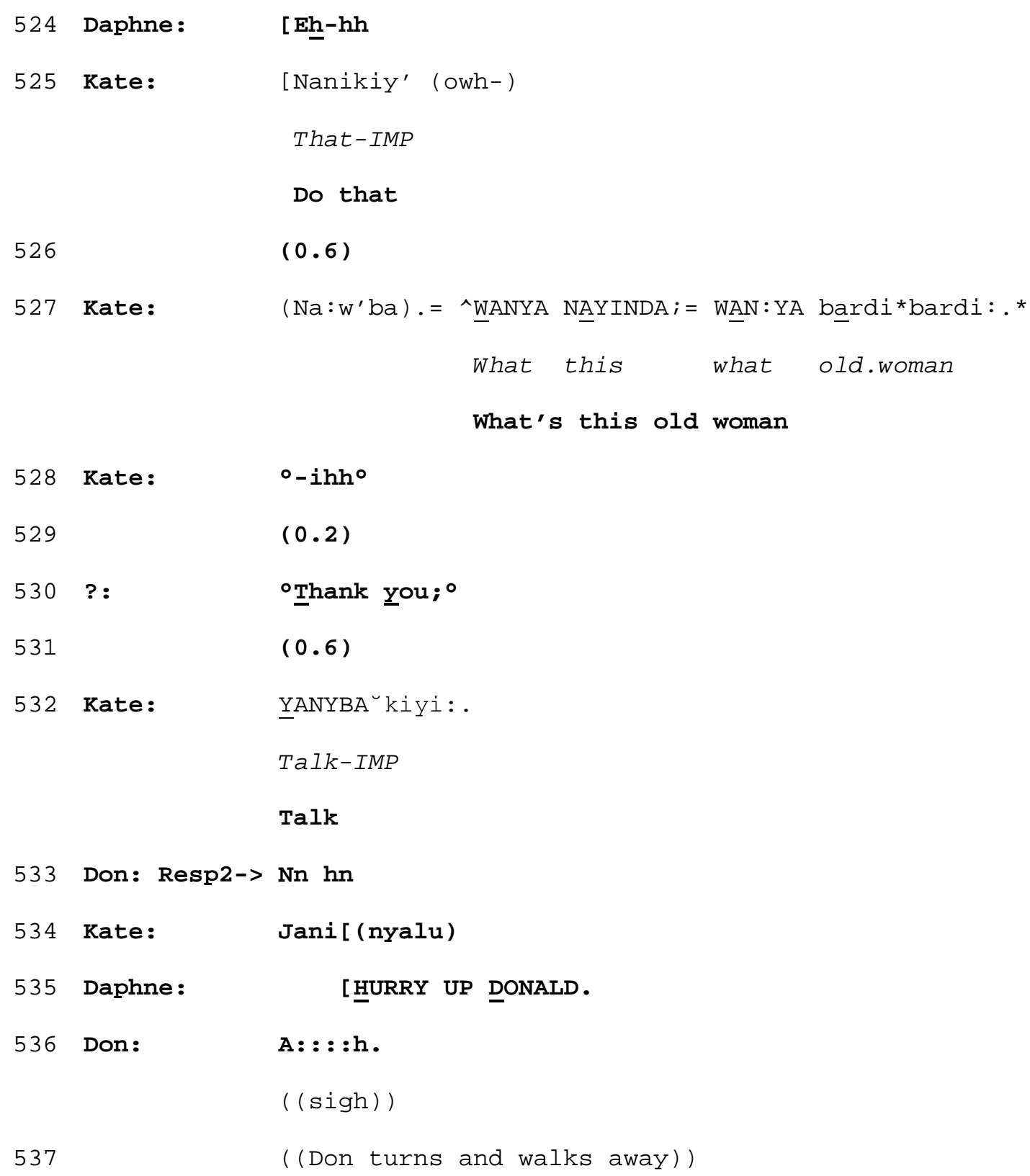

Kate and Daphne pursue the answer vigorously: Daphne through asking the question in different formats first in lines 499/502, and the redone in lines 506 and 508 (including stating the man's kin relationship, 'great uncle' or father's mother's brother), with a fourth version providing a candidate answer in lines 515-6 (suggesting 'Blackfella Spring’). Kate also pursues an answer by providing prompts ('He was here this morning', line 501/4), asides ('Ronnie did (see him)', line 511), clues ('Big mob of men still living there’, line 518), and directing him to go and find the answer from 'this old woman', (lines 520/22 and 
527). Don, however, remains almost silent throughout this barrage of questions and answer pursuits, apart from an indistinct 'wharah' (possibly 'where' in line 513), and an acknowledging 'nn hn' (line 533) and a sigh (line 536) just before he turns to walk away. An answer never comes from him. This is despite the fact that Daphne (especially) and Kate are, in Stivers and Rossano’s (2010) terms, strongly mobilising a response. Daphne uses interrogative syntax, there is a display on her part of an understanding that the man has epistemic rights to the knowledge, and she is gazing at him: three of the four response mobilizers. According to Stivers and Rossano, the presence of one mobilizer was enough to elicit a response in all 336 requests for information in their data set. Against this, in the early part of this sequence, Don was otherwise occupied with looking through the camera's viewfinder; second, he appears not to have the required knowledge for an answer (though, interestingly, he does not declare a lack of knowledge); and third, one could argue that his quiet 'nn hn' (533) is in fact a response: not an answer, but a response fitted to Kate's final injunction that he go and talk to 'the old woman'.

Like the non-Aboriginal interviewers that Eades (2007) reports on, Kate and Daphne are experiencing difficulty in eliciting information from this man. However, Kate and Daphne are Aboriginal themselves, and they are showing few signs of indirection in their questioning: perhaps because they are Elders, and because the information they are seeking is not sensitive. One final point: despite there being no answer, and very little verbal engagement from the question recipient here, there is abundant visual evidence from the video that Don is very much engaged with the question. He had been standing behind the camera, but just after he says 'Wharah' (line 513) he walks in front of the camera, and stands in front of the two seated women looking directly at them.

In the final example, not only is an answer (or a response of any kind) not forthcoming, but there is also very little evidence for engagement with the question by the question 
recipients, despite them (Daphne and Kate) being clearly within earshot, and Daphne being visually in line with Hilda. This sequence comes a little after the sequence in extract (10). Hilda is asking who the people at Blackfella Spring are, perhaps referring to the 'mob of men still living there' that Kate mentions in line 518 of extract (10).

(11) Porch:2.6:563

563

564

Hilda: Wanja'la; $=\left[\right.$ Blackfella Spring; $={ }^{\circ}$ wanya ${ }^{\circ}$, Who $3 p 1$ who

Q1a-> Who (are) they (at) Blackfella Spring, who, [((Gas escaping from DG's soft drink bottle $))$

565 $(7.7)$

566

567

568

569

570

Daphne:

$$
\text { 1SgACC SUn-ERG }
$$

The sun is burning me

571 Hilda:

[WANYA NANDA- ] =

What that

572 Hilda: $\mathrm{K}^{\prime} \mathrm{YAG}^{\prime} \mathrm{N} ;=$ KARRURRU.

many. thing east.place

Q1c-> What's all that in that place in the east

573

$$
(0.6)
$$


Hilda: [Whad dey call [dat.

Q1c-> What do they call that

575 Kate:

[M:argie $\smile(r)$ oughta ^lock dat-; (0.5)

576

ga:me up; you know?

Margie ought to lock that game up, you know

577

$(0.8)$

578 Kid:

'ere Nan

Here Nan

((Passes cup to Kate))

579

$(1.0)$

580 Kate :

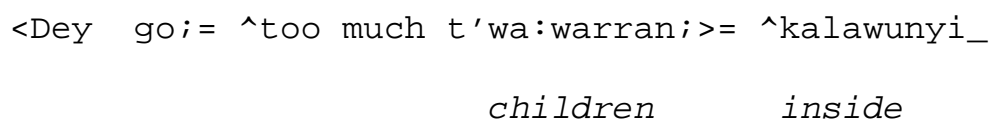

They've got too many kids inside

Hilda begins by asking who the people are at Blackfella Spring (line 564). This is followed by a yawning gap of 7.7 seconds. The video shows that Kate is engrossed in brushing her hair, and Daphne is looking down at her soft drink bottle as she lets gas out of it. Thus the lack of response could be attributed to 'activity occupied withdrawal' (Goodwin, 1981). On the other hand, neither activity is of a type that requires intense concentration: it is possible to talk whilst brushing one's hair, and it is also possible to talk whilst fiddling with the cap of a soft drink bottle. In fact, there is momentary engagement from Daphne when she shoots a glance over to Hilda as she says 'Spring' in line 564, which is at the transition relevance place. This suggests she has at the very least heard that Hilda is talking. Hilda's question is delivered relatively quietly, and has to compete with the sound of the gas escaping from Daphne's bottle. Be that as it may, there is no visible reaction from Kate, who continues her self-grooming, and Daphne returns her gaze to her drink bottle as she slowly lets the gas escape. After 7.7 seconds, Hilda asks a second, probably related question (line 566) about a place in the east. Again, Daphne looks over 
towards Hilda as she is finishing her question, showing that she has heard this talk too, but during the 2.6-second silence in line 567 she looks back down at her bottle and continues to screw the cap. Kate continues to groom. Hilda, who is now looking straight at Daphne, reinforces her question in line 568 by specifying more closely the location of the place she is talking about. After another 1.6 seconds of silence, Daphne remarks on her discomfort from the hot sun (570), which effectively ignores the question. However, Hilda does not give up, and overlapping the latter part of Daphne's complaint, she makes a third attempt at asking a question: rephrasing her question about the name of the place in the east (571-2/4). Kate is now finishing her grooming, and finally says something, but it has nothing to do with Hilda's question: she has some advice (or a complaint?) about a game that ought to be locked away. A boy then appears on the veranda to offer Kate a drink of water, and Hilda abandons her line of questioning. In the remainder of the recording, which goes on for another 11 minutes, they do not return to the questions. So here we have a question that is ignored. There is very little engagement with the question (none from Kate, and two brief glances from Daphne), despite the rather vigorous mobilizing pursuit of a response from Hilda. She has used interrogative syntax and gaze towards Daphne, but without success in attracting a response. The lack of a response is of course itself an action of sorts: a silent refusal to answer? Is this a case of what Eades (1985) refers to as the absence of obligation to give information, or lack of obligation on the part of the addressee to answer a question in Aboriginal society? In extract (10), Don did not attempt to provide an answer for which he appears not to have had the information to do so. In (11), there is not even anything but the weakest visual (and no verbal) engagement. It is at least possible that Kate's and Daphne’s engagement with their hair and soft drink bottle respectively can, in part at least, explain the lack of response. This, of course, does not invalidate Eades' claims. The database for this paper is small. Nevertheless, there are only two cases amongst 62 
question-answer sequences that show what amounts to declining to respond. There are a few other question-answer sequences in which reasons can be mustered for why no response was forthcoming. There are also numerous cases in which a response comes late, at least when cmpared to reports in the literature on second pair part 'delay'. However, most of the question-answer sequences in this collection follow a trajectory and structure that do not differ in any fundamental respect from what has been reported for other cultures or languages (cf. especially Stivers, Enfield and Levinson, 2010).

\section{Conclusion}

Extract (11) may be an example of the phenomenon that Walsh (1997:4) recounts:

So imagine a situation where I am asking: 'That green frog over there in the tree, have you got a name for that one?' pointing to a tree and pointing to a real frog that is right there - in front of us. What happens? Not only does the person not give me the answer. He looks away. There is no response whatsoever. There is just complete silence. Perhaps I have first asked that question on a Tuesday. When I am down at the camp again on Wednesday of the following week back comes the answer. The man says: 'That one, that green one there, we call than one durket.' When he says this without any kind of explicit linkage to the previous questioning session, it is already over a week. So I ask: 'What is this green one?' Perhaps wondering whether I am a little slow, he says: 'You know, in the tree.' And I wonder if he is referring to a leaf. 'No, green one, in the tree, durket.' Eventually it dawns on me that he is referring to the green frog that I asked about over a week ago...So this is what I am calling a 'delayed' reaction. From the 
perspective of the Aboriginal speaker there does not seem to be any particular delay at all. It is from the perspective of the Anglo speaker that there is a 'delay'

There is no getting around the need for more evidence than is presented from the question-answer sequence data I am presenting here. However, most questions are responded to, and only a minority are not, most of which can be accounted for. Where an answer is missing, in almost all cases the questioner pursues an answer. Furthermore, the data is this collection is Indigenous conversation, not talk between Indigenous Australians and non-Indigenous Australians. However, it is striking that most question-answer sequences follow the format of what is known about question-answer sequences in other languages and cultures. Compared to the 10 languages that are reported on in the Max Planck project (Stivers et al. 2009; Stivers, Enfield and Levinson, 2010), the mean delay of the answers in this Garrwa talk is long: at just under 0.8 seconds it is more than half as long again as the longest in their 10 languages. Second, the proportion of questions that are not followed by a relevant response is, at $26 \%$, about the same as Lao, the language which has the highest proportion of questions with no response in that collection; and the proportion of questions that are not followed by an answer, at $45 \%$, is considerably higher than for Korean, the language with the highest proportion in that collection. There are also a few questions that never received an answer (at least not during this conversation), and the reasons may not be because the recipients have not heard, or do not have rights to answer, or lack of gaze, or hesitancy in the environment of a dispreferred response. In extract (11), we saw evidence that Daphne had heard at least some of Hilda's series of questions (as probably had Kate). She had rights to answer. The topic was not a taboo one, as far as we can ascertain. This occurred despite Hilda's questions having three of the features that 
Stivers and Rossano (2010) found in their data set to more or less guarantee the mobilization of a response.

There is no evidence that either the fundamentals of turn-taking as described in Sacks, Schegloff \& Jefferson (1974), or the fundamentals of adjacency pair organisation as described in Schegloff \& Sacks (1973) or Schegloff (2007) are any different in these data set used for this study. These speakers overwhelmingly speak when selected, and mostly provide fitted responses as second pair parts when questioned. There is, however, some evidence that gaps are not always minimized, and that answers (as second pair parts) do not always come directly after the question, even in cases in which there is no evidence of dispreference in the answer, of 'activity occupied withdrawal' (Goodwin, 1981) on the part of the question recipient(s), or of some other trouble in the talk. These latter observations may be indicative that the speakers in these conversations are in a 'continuing states of incipient talk’ (Schegloff \& Sacks, 1973). The women grew up together, so are more like family than close friends (in fact, Hilda and Daphne are sisters-in-law); they have known each other and lived in small communities in close proximity to each other for many years. Extended silences between turns - unminimized gaps that do not transform into lapses - are not only tolerated, but may even become the norm in the more 'unfocused' or unconstrained phases of their interactions. In addition, these women sit around for hours at a time, passing much of the day sitting outdoors engaged in slow and leisurely conversation, and observing the passing of events and people from their porch. When questions are asked, an answer can still be seen to be conditionally relevant in the vast majority of cases, and sometimes these answers are pursued insistently, even vigorously (especially perceptible in extracts (10) and (11)). On the occasions when no response comes, it is rare for the recipient not to orient to the question, and on such cases when they do not, it may in fact be the speakers ways of indicating that they do not have the 
knowledge to provide an answer. Long gaps between first and second pair parts, and occasional non-responses, may, as Couper-Kuhlen (2010) puts it, 'be one way in which copresent parties construct an encounter as nonfocused' (p. 35). That is, the data presented in this paper may provide examples of interactional conduct found in 'nonfocused gatherings and incipient states of talk’ (p. 36).

\section{References}

Brown P 2010 'Questions and their responses in Tzeltal’ Journal of Pragmatics 42: 26272648.

Couper-Kuhlen E 2010 ‘Commentary on Stivers and Rossano: "Mobilizing Response” Research on Language and Social Interaction 43: 32-37.

Eades D 1985 'You gotta know how to talk . . . information seeking in South-east Queensland Aboriginal Society’ In J Pride (ed) Cross-cultural Encounters: Communication and Mis-communication. River Seine. Pp. 91-109.

Eades D 1992 Aboriginal English and the Law: Communicating with Aboriginal English Speaking Clients: A Handbook for Legal Practitioners Brisbane: Queensland Law Society.

Eades D 1993 'The case for Condren: Aboriginal English, pragmatics and the law Journal of Pragmatics 20: 141-162.

Eades D 2007 ‘Understanding Aboriginal silence in legal contexts’ In H Kotthoff \& H Spencer-Oatey (eds) Handbook of Intercultural Communication. Berlin: Mouton de Gruyter. Pp. 285-301.

Enfield NJ 2010 ‘Questions and responses in Lao’ Journal of Pragmatics 42: 2649-2665. 
Englert C 2010 'Questions and responses in Dutch conversations Journal of Pragmatics 42: 2666-2684.

Gardner R \& I Mushin 2007 ‘Overlapping talk in a Garrwa community’ Australian Review of Applied Linguistics 30: 35.1-35.14.

Goffman E 1963 Behavior in Public Places New York: The Free Press.

Goodwin C 1981 Conversational Organization New York: Academic Press.

Hoymann G 2010 ‘Questions and responses in $\$ \bar{A}$ khoe Haill om’ Journal of Pragmatics 42: 2726-2740.

Kendon A 1988 ‘Goffman’s approach to face-to-face interaction’ In P Drew \& A Wootton (eds) Erving Goffman: Exploring the Interaction Order Cambridge: Polity Press. Pp. 14-40.

Levinson S 2006 'On the human “interaction engine”' In N Enfield \& S Levinson (eds) Roots of Human Sociality Oxford: Berg. Pp. 39-69.

Levinson S 2010 'Questions and responses in Yélî Dnye, the Papuan language of Rossel Island’ Journal of Pragmatics, 42: 2741-2755.

Mushin I and R Gardner 2009 'Silence is talk: Conversational silence in Australian Aboriginal talk-in-interaction’ Journal of Pragmatics 41: 2033-2052.

Raymond G 2003 'Grammar and social organization: Yes/no interrogatives and the structure of responding 'American Sociological Review 68: 939-967.

Sacks H, E Schegloff \& G Jefferson 1974 'A simplest systematics for the organization of turn-taking for conversation`Language 50: 696-735.

Schegloff E 1968 'Sequencing in conversational openings' American Anthropologist 70: 1075-1095.

Schegloff E 1996 'Confirming allusions: Toward an empirical account of action` The American Journal of Sociology 102: 161-216. 
Schegloff E 2006. 'Interaction: The infrastructure for Social Institutions, the natural ecological niche for language, and the arena in which culture is enacted' In N Enfield \& S Levinson (eds) Roots of Human Sociality Oxford: Berg. Pp. 70-96.

Schegloff E 2007 Sequence Organization in Interaction: A Primer in Conversation Analysis Cambridge: Cambridge University Press.

Schegloff E 2010 'Commentary on Stivers and Rossano: “Mobilizing Response”’ Research on Language and Social Interaction 43: 38-48.

Schegloff E \& H Sacks 1973 ‘Opening up closings‘ Semiotica 8: 289-327.

Sidnell J 2001 ,Conversational turn-taking in a Caribbean English Creole‘ Journal of Pragmatics 33: 1263-1290.

Stivers T 2010 'An overview of the question-response system in American English conversation’ Journal of Pragmatics 42: 2772-2781.

Stivers T, N Enfield, P Brown, C Englert, M Hayashi, T Heinemann, G Hoymann, F Rossano, JP de Ruiter, K-E Yoon \& S Levinson 2009 ‘Universals and cultural variation in turn-taking in conversation' Proceedings of the National Academy of Sciences 106: 10587-10592.

Stivers T, N Enfield \& S Levinson 2010. 'Question-response sequences across ten languages: An introduction’ Journal of Pragmatics 42: 2615-2619.

Stivers T \& F Rossano 2010 ‘Mobilizing Response’ Research on Language and Social Interaction 43: 3-31.

Walsh M 1997 ‘Cross cultural communication problems in Aboriginal Australia’ North Australia Research Unit Discussion Paper 7/1997 Canberra: ANU. Pp. 1-23. 


\section{Glossary}

ABL - ablative

ACC - accusative

BARRI - a discourse particle

CONJ - conjunction

DAT - dative

ERG - ergative

HAB - habitual

IMP - imperative

LOC - locative

NA - a discourse particle

PA - past tense

PART - particle

PL - plural

PRES - present tense

$\mathrm{Br}$ - brother

Fa - father

Mo - mother

1sg - first person singular

2du - second person dual

2sg - second person singular

3du - third person dual

3pl - third person plural 
${ }^{\mathrm{i}}$ This is not to say that there is a denial in CA work that cultural differences exist, but that these differences play out through variations in practice of these fundamentals. For example, it would appear that in some cultures compliments are generally rejected by compliment recipients, whilst in others they are more likely to be downgraded or accepted. However, in all cases the recipients are dealing with the compliment, and it would be very rare (and subject to special contextual considerations) for a compliment to be responded to by, for example, an insult or a greeting.

ii This contrast strongly with the Stivers et al. (2009) study of question-answer sequences in ten languages, in which polar questions were found to be the commonest type in nine of the languages.

iii Of the eight polar questions, seven are in Kriol, and none of these has polar question syntax (i.e. auxiliary verb inversion): and six are declarative questions with rising terminal intonation (the seventh is a terminally falling declarative). The eighth example is a rare case of the use of 'Kuna' (Ilana Mushin, pers. comm.), which is a polar question particle. Interestingly, this utterance has falling terminal pitch, similar to most English WH-question utterances. It is also striking how few polar questions there are in the data set, even though I undertook a second careful search for examples on the suggestion of one of the reviewers. ${ }^{\text {iv }}$ Comparisons with the Stivers et al. (2009) are constrained by that study being limited to polar questions. It is nevertheless striking that on several of the parameters, these Garrwa conversations lay outside the range of results for those ten languages: offset times for responses were longer than for any of those languages, the proportion of questions not receiving an answer was higher, and the proportion of questions receiving no response at all was higher (with the exception of Lao, which was similar).

${ }^{v}$ All names are pseudonyms.

${ }^{\mathrm{vi}}$ In the interests of readability of the transcripts, notation of non-verbal activity has been kept to a minimum, as the transcripts are already fairly unwieldy with glossing and translations. The downside is that the reader is required to do more work to locate reference to non-verbal activity in the discussion. 